\title{
INTEGRACIÓN DE VISUALIZACIÓN CIENTÍFICA MOLECULAR EN EL SALÓN DE CLASES
}

\author{
Miguel Á. García-Ruiz* \\ Facultad de Telemática, Universidad de Colima, C.P. 28040, Colima, México \\ Laura L. Valdez-Velazquez y Zeferino Gómez-Sandoval \\ Facultad de Ciencias Químicas, Universidad de Colima, km 9 Carr. Colima-Coquimatlán, Colima, México
}

Recebido em 18/9/07; aceito em 12/6/08; publicado na web em 10/11/08

\begin{abstract}
INTEGRATION OF MOLECULAR SCIENTIFIC VISUALIZATION IN THE CLASSROOM. For some years, Chemistry teachers have used scientific visualization software of molecular models in computing rooms and chemistry laboratories for educational purposes. However, its application in classrooms has been limited. This article describes the integration and use of computer programs for scientific molecular visualization in a traditional classroom. We consider that the improvement of technical aspects of their application and use (usability) has a direct effect on students' understanding of molecular structures (including students' extrinsic motivation), among other factors. Consequently, we developed a guide for the integration of hardware and software of molecular visualization for its use in the classroom.
\end{abstract}

Keywords: molecular; visualization; classroom.

\section{INTRODUCCIÓN}

Los modelos de las estructura moleculares principalmente en la bioquímica son considerados complejos por la mayoría de los estudiantes de química, ya que involucran múltiples agrupaciones de átomos en las moléculas.

Dentro del campo de la bioquímica, las proteínas son biomoléculas essenciales para el funcionamiento de la vida. Son macromoléculas complejas construidas de aminoácidos que se acoplan con otras proteínas o con ADN (ácido desoxirribonucleico) generando estructuras muy complejas con funciones particulares de acuerdo a su plegamiento, como el reconocimiento molecular, el transporte de moléculas, la catálisis de las reacciones químicas, así como actividades relacionadas con la regulación génica. Esto nos conlleva a considerar la importancia de conocer y entender la relación estructura-actividad a nivel molecular de las proteínas. Los argumentos anteriores nos conducen de manera natural a la utilización de modelos moleculares para comprender los fenómenos químicos y biológicos. ${ }^{1}$ El uso de los modelos moleculares no solo ha impactado en la investigación científica sino también en la enseñanza de la química, incluso en 1811 Dalton ya los utilizaba en sus cátedras. ${ }^{2}$

Hace varias décadas, los únicos modelos para el aprendizaje de las estructuras de las moléculas biológicas consistían en descripciones e ilustraciones en libros. Posteriormente, a principio de 1930, se utilizaron modelos comerciales denominados de space filling de Stuart en versión de plástico y de madera para representar estructuras moleculares. ${ }^{3}$ Sin embargo, para grandes estructuras involucra un gran costo, además que tienen una gran rigidez para mostrar movimientos o plegamientos y no representan las interacciones con otras moléculas, estos son modelos que pierden al alumno en su imaginación. ${ }^{4}$ El uso de estos modelos confirma la importancia de contar con distintas técnicas de visualización molecular. De hecho en el pasado el término visualización era sinónimo de imaginación y actualmente ésta palabra está más relacionada con las imágenes que mejoran nuestra imaginación y experiencia visual. ${ }^{5}$

Un método más versátil para estudiar estructuras moleculares

\footnotetext{
*e-mail: mgarcia@ucol.mx
}

biológicas en tercera dimensión, es por medio de sofware de visualización científica molecular. Estos son programas con interfaces gráficas capaces de proyectar estructuras tridimensionales que facilitan enormemente la interacción humano-computadora. El uso de esta tecnología por los docentes en el salón de clase es cada día más común, principalmente en los cursos de química. ${ }^{6,7}$

El objetivo de la visualización científica es mostrar a los usuarios de manera eficiente, clara y entendible los datos obtenidos de fenómenos o experimentos científicos, principalmente por medio de imágenes o grafos a través de una interfaz gráfica de alto desempenho. ${ }^{8}$

Hasta hace unos pocos años, si se deseaba utilizar la visualización molecular era necesario correr programas en grandes módulos de computadoras y dicho software era especializado, no disponible para los estudiantes. En esos años no era posible usar una laptop y obtener por el Internet software gratis. Hoy en día, estos programas son obtenidos libremente en la red y su instalación y uso es fácil. Ahora, el aprendizaje asistido por la visualización científica molecular está cambiando la manera en que los estudiantes conciben la química. ${ }^{9}$ En un proyecto de investigación cooperativa financiado por la Fundación de la Ciencia Nacional de los Estados Unidos, sobre la visualización molecular en la enseñanza de la química, se encontró que de todos los modelos moleculares considerados, incluyendo los modelos físicos, las herramientas de visualización computacional fueron los que marcaron la diferencia en la manera en que los alumnos asimilan el conocimiento. ${ }^{10-15}$

La visualización científica molecular se ha utilizado como una herramienta valiosa en el aprendizaje de la química en el salón de clases. ${ }^{16-20}$ Hay muchos programas disponibles que permiten al alumno manipular y entender modelos de moléculas pequeñas y grandes (macromoléculas). Por varios años el programa más popular para gráficos moleculares ha sido el RasMol, el cual fue desarrollado por Roger Sayle en la Universidad de Edimburgo, Reino Unido, a mediados de los años $90 .{ }^{21}$ Poco después fue actualizada y se puede descargar de su sitio Web. ${ }^{22,23}$ La versión de RasMol para el sistema operativo Windows se ha denominado Raswin (mostrado en la Figura 1), siendo la versión 2.7 la más actual a la fecha, y se puede descargar de manera gratuita. ${ }^{23}$ Cabe hacer notar que se ha desarrollado una versión en español del manual de RasMol. ${ }^{24}$

Otro programa de visualización molecular gratis disponible en 


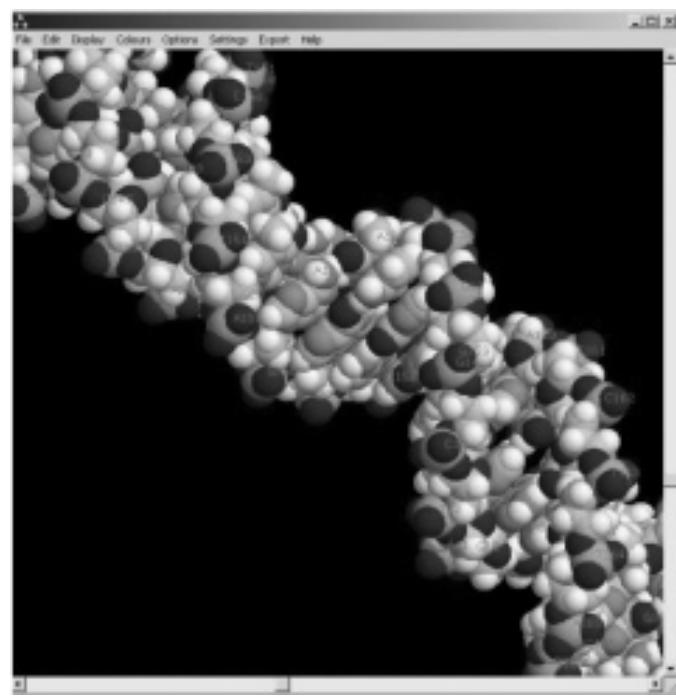

Figura 1. Programa Raswin desplegando un fragmento de una cadena de $A D N$

Internet es el Protein Explorer desarrollado por Eric Martz, el cual ofrece una interfaz basada en el RasMol pero es implementado en Chime, un plug-in para páginas Web. ${ }^{25}$ Otro programa popular es el Deep View (Swiss-PDB Viewer) y el Mage. ${ }^{26,27}$ Existen una gran variedad de programas para la visualización de proteínas y van desde aquellos que son de dominio público (su obtención es libre de costos), hasta algunos que requieren licencias académicas, para educación o investigación. En la Tabla 1 se presenta una lista de algunos de estos programas así como algunas de sus características.

Recientemente, se ha publicado en la literatura una herramienta de visualización molecular denominada jAMVLE (por las siglas en inglés de java- based jmol Amalgamated Molecular Visualization Learning Environment) usada en la enseñanza y aprendizaje de estructuras moleculares dentro del ramo de la bioquímica, biología molecular, química orgánica e inorgánica, cristalografía y ciencias de los materiales. ${ }^{28}$ Este programa puede descargarse gratuitamente. ${ }^{29}$

También en el 2006 se publicó las bondades y ventajas de un nuevo visualizador denominado BALLView utilizado en la enseñanza de la bioquímica y la bioinformática. ${ }^{30}$ Es un programa que corre en la mayoría de las plataformas (Windows, MacOS X y Linux) y puede descargarse gratuitamente. ${ }^{31}$
Tabla 1. Programas para la visualización molecular, utilizados ampliamente en la academia

\begin{tabular}{|c|c|}
\hline Nombre del visualizador & Características \\
\hline Chime & Se opera en línea a través del Internet. \\
\hline Chimera & $\begin{array}{l}\text { Características básicas para } \\
\text { construir modelos estructurales. }\end{array}$ \\
\hline RasMol & Visualización de estructuras complejas. \\
\hline PyMol & $\begin{array}{l}\text { Visualización molecular } \\
\text { sencillas y complejas. }\end{array}$ \\
\hline VMD & $\begin{array}{l}\text { Visualización científicas y análisis } \\
\text { estructural, con énfasis } \\
\text { en problemas dinámicos. }\end{array}$ \\
\hline
\end{tabular}

\section{CARACTERÍSTICAS Y FUNCIONALIDAD DEL RASMOL}

Consideramos que el RasMol es un programa de visualización molecular versátil y es excelente para explicar o demostrar conceptos básicos de estructuras moleculares en sus diferentes representaciones (ball-and-stick, CPK o wireframe). Además, es capaz de desplegar modelos de macromoléculas como la Troponina o el ADN, sin necesidad de utilizar una computadora de escritorio o laptop poderosa para su aplicación en el salón de clases. En nuestra opinión, RasMol tiene ciertas ventajas y desventajas con respecto a otros visualizadores, las cuales muestran en la Tabla 2.

Básicamente lo que hace RasMol es leer un archivo que contiene la información estructural de la molécula, como por ejemplo un archivo en formato PDB (Protein Databank) y presentar en pantalla el modelo gráfico, como resultado de la localización de cada uno de los átomos de la molécula, sobre un plano de coordenadas cartesianas $x, y, z$ y de la construcción de un gráfico que relaciona las entidades que componen el sistema.

EL PDB es un formato estándar para representar datos estructurales (en 3D) de moléculas, especialmente proteínas y macromoléculas biológicas, el cual almacena principalmente coordenadas atómicas ortogonales y los respectivos elementos químicos que componen la molécula. ${ }^{32}$ Una vez que RasMol presenta la estructura, permite manipularla mediante un conjunto de sencillos comandos que dan la posibilidad desde seleccionar un átomo hasta dejar una cadena titilando con el fin de hacer más evidente una explicación determinada.

Tabla 2. Ventajas y desventajas del programa RasMol

\begin{tabular}{l}
\hline Ventajas \\
\hline Existen versiones para muchos sistemas operativos, \\
tales como Unix, Linux, Macintosh y Windows. \\
Existe mucho soporte en la Web, se pueden encontrar \\
manuales, guías de práctica, sugerencias, etc. \\
Las visualizaciones pueden ser programables \\
(el RasMol maneja scripts). \\
Es gratuito para aplicaciones educativas
\end{tabular}

Es muy fácil de instalar, requiere relativamente poco poder de cómputo aún para visualizar macromoléculas complejas, es un programa relativamente pequeño pero poderoso.

Permite generar imágenes de alta calidad de modelos moleculares, para utilizarse en publicaciones de revista o libros.

Permite observar la molécula en modos CPK, wireframe y otros, además de mostrar etiquetas (iniciales de los elementos de cada átomo y otras)

\section{Desventajas}

Los scripts pueden ser difíciles de programar.

Solamente muestra una molécula a la vez.

No se visualiza el archivo de texto PDB (Protein Databank).

No tiene enlaces con otras herramientas de biología molecular o páginas Web.

El centrado y acercamiento de la molécula puede ser difícil de realizar.

No permite funciones avanzadas que están presentes en otros visualizadores, como el despliegue de mapas de densidad de electrones. 
Pero va incluso más allá, permite también la creación de scripts o animaciones moleculares, los cuales permiten mostrar transformaciones sucesivas sobre una molécula ...imagine por ejemplo la doble hélice del ADN desenrollándose para explicar el proceso de la replicación ante sus ojos... o por ejemplo, cambiar la perspectiva de visualización a través de comandos de rotación, translación o cambio de tamaño de la molécula en la pantalla; colorear y etiquetar los residuos de interés de manera específica. Existen colores estándar para cada uno de los tipos de átomos: verde para el carbono, rojo para el oxígeno, azul para el nitrógeno, blanco para el hidrógeno, amarillo para el azufre, sin embargo el usuario puede cambiar el código de colores. Otras de las funciones que se pueden utilizar son las determinaciones geométricas entre residuos (distancias y ángulos), la representación del volumen de los átomos, así como realizar indicaciones sobre la superficie de la molécula, ya sea en forma de malla o bien sólida o transparente.

El uso de esta clase de herramientas computacionales en el salón de clases está aún muy poco explotado en Latinoamérica, se tienen desarrollos hechos, pero no se tiene ninguno que sea coherente y que esté implementado dentro de un programa real de pregrado de bioquímica o biología molecular. Las posibilidades que el uso de esta clase de software son muy amplias pues permiten la participación activa del estudiante dentro de su proceso educativo y demandan un mayor nivel de exigencia pedagógica para el docente, esto convertiría una clase tediosa a una clase interactiva y emocionante. Los desarrollos educativos, en este sentido se realizan relativamente a bajo costo, el mayor obstáculo lo constituye la falta de conocimiento de las posibilidades pedagógicas de estas herramientas; y la ausencia de compromiso, entre los miembros la comunidad universitaria, frente a la innovación que implica el desarrollo de un proceso educativo.

Este artículo describe aspectos prácticos de integración y uso de un visualizador molecular como el Raswin en el salón de clases. Para analizar el uso de un programa de computadora se tienen que llevar a cabo estudios de usabilidad. La usabilidad analiza principalmente la eficiencia, eficacia, seguridad y satisfacción de uso de una interfaz de computadora. ${ }^{33}$ Es bien sabido que si un programa de computadora educativo es muy utilizado, éste tendrá un soporte más adecuado para el aprendizaje. ${ }^{34}$ En definitiva, las herramientas de software para bioinformática se han utilizado de manera muy eficaz y eficiente. ${ }^{35}$ La motivación que tendrán los alumnos al seguir utilizando el programa de visualización molecular es otro de los aspectos que estudia la usabilidad.

Existen métodos estandarizados para llevar a cabo pruebas de usabilidad. Uno de ellos es la aplicación de cuestionarios con preguntas de actitud y de opinión y escalas tipo Likert, los cuales se aplican como post-test. ${ }^{33}$ La ventaja de aplicar cuestionarios de usabilidad es que se obtienen datos cuantitativos y cualitativos con retroalimentación importante desde el punto de vista de los usuarios de un software (en este caso los alumnos de química), además de ser de bajo costo. La desventaja de los cuestionarios es que pueden estar limitados a no medir ciertos datos cuantitativos de mucho valor, como la duración de la prueba de usabilidad o recurrencia de los usuarios en algunas tareas de la prueba. ${ }^{36}$

\section{ESTUDIO EXPERIMENTAL DE USABILIDAD}

Habiendo descrito lo anterior, diseñamos un estudio exploratorio acerca de la usabilidad del Raswin en un salón de clases tradicional, donde establecimos las siguientes preguntas de investigación: 1. ¿La calidad de proyección de las gráficas puede afectar la percepción general de aspectos importantes de las estructuras moleculares? 2. ¿Los estudiantes encontrarán los comandos del Raswin fáciles de usar en clases? 3. ¿El uso de un visualizador molecular como el Raswin motivará a los estudiantes a seguir utilizando visualizadores moleculares en subsiguientes clases?

\section{Metodología}

\section{Diseño}

El estudio piloto se llevó a cabo en uno de los salones de clase de la Facultad de Ciencias Químicas perteneciente a la Universidad de Colima, México. El salón tiene una capacidad para unos 40 estudiantes, con ventanales grandes a los costados, los cuales tienen persianas para obscurecer el salón cuando se utiliza el cañón de proyección, el cual se encuentra instalado al centro del techo, a una distancia aproximada de $3.5 \mathrm{~m}$ de la pared frontal donde se realizan proyecciones, sobre una pantalla enrollable que mide aprox. $1.50 \mathrm{~m}$ de ancha (Figura 2) Sin embargo, los profesores generalmente proyectan sobre un pintarrón de color blanco que se encuentra instalado sobre esa pared, el cual mide aprox. $4 \mathrm{~m}$ de ancho y $1.20 \mathrm{~m}$ de alto. Tanto los estudiantes como el profesor se encuentran a la misma altura, no hay estrado o gradas alguna en el suelo del salón de clases.

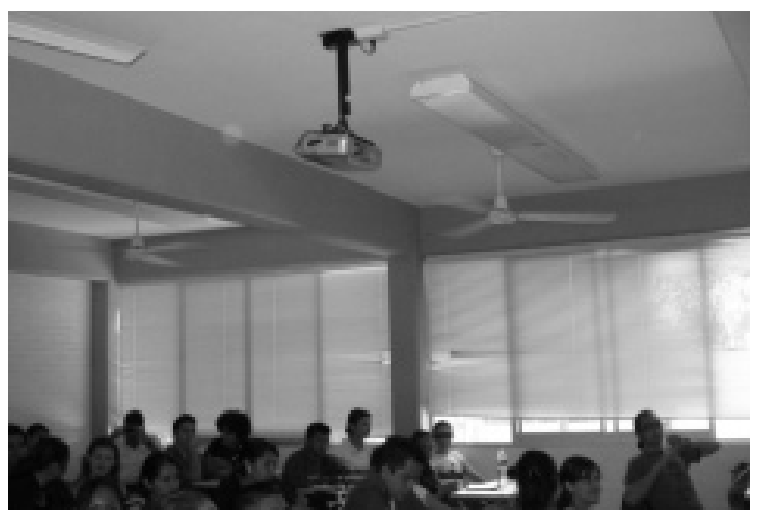

Figura 2. Fotografía del salón de clases, donde se muestra los ventanales grandes a los costados, los cuales tienen persianas para obscurecer el salón y el cañón de proyección, en el techo

Ya que se buscó estudiar las diferencias de percepción visual y motivación de los estudiantes con respecto a la proyección con dos cañones y superficies diferentes de un modelo molecular, se dividió el grupo en dos subgrupos (A y B), asignando a los estudiantes a cada subgrupo de manera aleatoria. Con el primero (grupo A) se utilizó el equipo que ya estaba instalado en el salón, mientras que con el B se utilizó equipo de proyección que se instaló en ese mismo salón de clases (Figura 3).

\section{Materiales}

Para el estudio piloto se utilizaron dos superficies de proyección y dos cañones de datos. Con el subgrupo A se utilizó el equipo que ya estaba instalado en el salón de clases: un cañón marca Nec, modelo VT465, con una resolución nativa de 800 x 600, un contraste de 400:1 y con 1800 lúmenes ANSI y el pintarrón de color blanco. El cañón estaba instalado en el techo al centro del salón, a una distancia de aprox. $3.5 \mathrm{~m}$ del área de proyección.

Con el subgrupo B se utilizó un cañón de datos marca Infocus, modelo LP600 con una resolución nativa de 1024 x 768, con un contraste de 1000:1 y con 2000 lúmenes ANSI. Este cañón fue instalado aproximadamente a $3.5 \mathrm{~m}$ del área de proyección, sobre una mesa pequeña situada al centro del salón de clases. La proyección se realizó sobre una pantalla portátil de color blanco mate especial para proyecciones de cañón, marca Alfra, modelo Silver Screen, de 1.80 x 1.80 mts., la cual se desplegó frente al pintarrón.

En ambas pruebas con los subgrupos se utilizó una computadora portátil marca Acer modelo Ferrari 3000, con 128 Mb de gráficas de video, $512 \mathrm{Mb}$ de memoria RAM, y con una velocidad de $3 \mathrm{GHz}$, en 


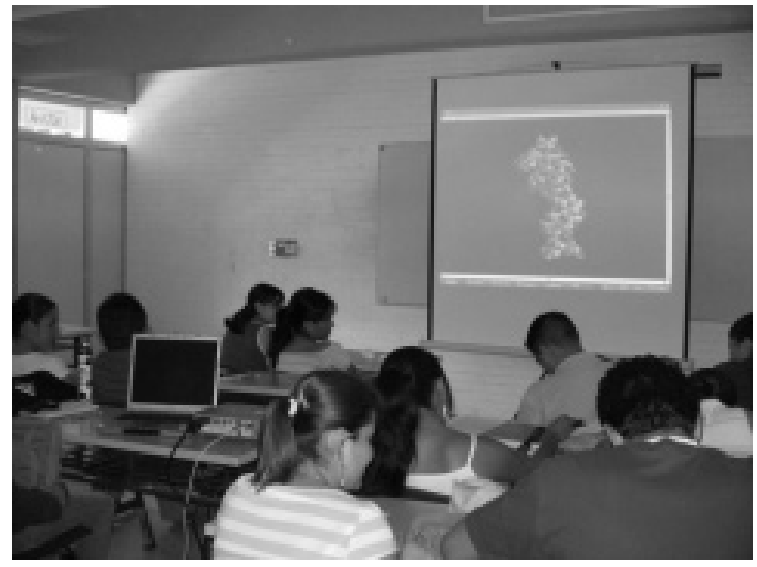

Figura 3. Fotografía donde se muestra la proyección con el grupo B en el mismo salón de clases, con un equipo portátil

la cual estaba instalado el programa Raswin versión 2.7.1. que se utilizó para mostrar la estructura molecular del ADN. Para obtener datos demográficos y de usabilidad (cualitativos y cuantitativos), se aplicó a cada estudiante un cuestionario post-test, editado especialmente para este estudio exploratorio, el cual se muestra en el Anexo A. Este cuestionario incluye escalas del cuestionario estándar de usabilidad SUS (System Usability Scale). ${ }^{37}$ El cuestionario aplicado en este estudio también incluye preguntas demográficas y de usabilidad estándares que se han manejado en otros cuestionarios de usabilidad del campo de la Interacción Humano-Computadora. Este cuestionario fue piloteado previamente con un grupo de 5 alumnos para evitar ambigüedades en el texto, aunque las escalas del cuestionario SUS han sido ampliamente validadas por el autor del mismo. ${ }^{37} \mathrm{El}$ modelo molecular de la molécula de ADN utilizado en este estudio es un archivo en formato PDB (Protein Data Bank), obtenido de la página de Glactone. ${ }^{38}$

\section{Participantes}

En esta prueba de usabilidad participaron 25 estudiantes del grupo de $6^{0}$ semestre de la carrera de Químico Farmacéutico Biólogo. Los participantes del subgrupo A (los que utilizaron el equipo del salón de clases) fueron 16 estudiantes ( 8 hombres y 8 mujeres) con una edad promedio de 20 años. Casi todos han jugado videojuegos con experiencia de muchos meses, y 12 de ellos han utilizado programas de visualización molecular. Ninguno de ellos ha utilizado el programa Raswin que se utilizó en este estudio. Solamente 2 estudiantes presentaron problemas de la vista (miopía). Los participantes del subgrupo B (los que utilizaron el equipo de proyección instalado ex profeso en el salón de clases) fueron 19 estudiantes ( 8 hombres y 11 mujeres) con una edad promedio de 21 años. Casi todos han jugado videojuegos con experiencia de muchos meses, y 9 de ellos han utilizado programas de visualización molecular. Solamente 2 estudiantes de este grupo han utilizado el programa Raswin por varios meses. Ocho estudiantes presentaron problemas de la vista (miopía y astigmatismo).

\section{Procedimiento}

El estudio de usabilidad duró aproximadamente 40 min para cada subgrupo, el cual se acerca a la duración de las clases impartidas en las aulas de la Facultad de Ciencias Químicas, que es de 50 min. Cada subgrupo por separado recibió una plática impartida por uno de los investigadores de este estudio. La plática consistió en que los alumnos analizaran sistemáticamente aspectos estructurales de la molécula de ADN, utilizando el programa Raswin. Un estudiante de cada subgrupo voluntariamente aceptó manipular el modelo gráfico del ADN en la computadora portátil, siguiendo instrucciones por parte del investigador que dio la plática, mientras que éste explicaba a los estudiantes los aspectos estructurales del modelo en la pantalla de proyección. Los aspectos estaban enmarcados en las siguientes preguntas dirigidas a los estudiantes, las cuales debieron responder al momento de visualizar la molécula: ¿Cuántas cadenas aparecen? ¿Dónde están los aminoácidos hidrofóbicos? ¿Dónde están las hélices alfa y las hojas ß? ¿Cómo puedo determinar la distancia entre dos átomos? ¿Cómo se puede ver el interior de la molécula? ¿Dónde están los puentes de hidrógeno?

Estas preguntas y sus instrucciones para las respuestas fueron extraídas y adaptadas de la página Web desarrollada por Gonzalo Claros. ${ }^{39}$

Para responder cada una de las preguntas anteriores, el alumno voluntario que utilizó la computadora portátil escribió una serie de instrucciones y seleccionó ciertas opciones en el menú del programa Raswin. Por ejemplo, para responder la primera pregunta, el alumno escribió las siguientes instrucciones, las cuales fueron dictadas por el investigador que condujo el estudio: Menu $->$ Display $->$ Backbone ; Menu $->$ Colour $->$ Chain. Dar click para identificar la letra asignada a cada cadena. Suponiendo que quieras ocultar todas las cadenas excepto las B y D: restrict :b or :d. Para volver a mostrar todas las cadenas: Menu Edit $->$ Select all

Para ver las demás instrucciones utilizadas en el estudio, consultar a Claros. ${ }^{39}$

Una vez terminadas las explicaciones utilizando el Raswin, se aplicó el cuestionario de usabilidad (mostrado en el Anexo A) a cada uno de los estudiantes.

\section{RESULTADOS}

Con respecto a los datos demográficos comparados con los resultados del cuestionario, no existe influencia alguna; los estudiantes que tenían experiencia previa considerable en videojuegos y con visualizadores moleculares respondieron aproximadamente igual que los demás estudiantes. Los estudiantes con problemas de la vista lo hicieron de igual manera.

El cuestionario de usabilidad generó datos cuantitativos (escalas de opinión) y datos cualitativos (preguntas abiertas).

Como se puede ver en la Figura 4, los resultados sugieren que la resolución del cañón y el tipo de pantalla utilizado no influenciaron

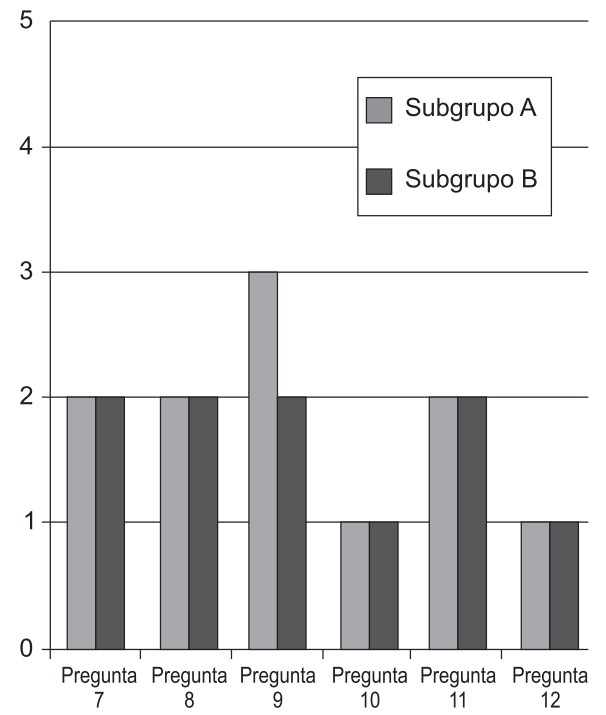

Figura 4. Resultados de las escalas del cuestionario de usabilidad. Valores de la escala: $1=$ muy fácil/totalmente de acuerdo...5=muy difícil/totalmente en desacuerdo 
los comentarios de los alumnos con respecto a la percepción de la tridimensionalidad, y la calidad visual del modelo molecular. Todos los estudiantes están completamente de acuerdo en seguir utilizando el programa en clases subsecuentes.

La mayoría de los estudiantes del subgrupo B (los que utilizaron el equipo que se instaló ex profeso) marcaron en la escala como "fácil" el uso de comandos (instrucciones) para la manipulación y análisis de la estructura molecular en el Raswin. Sin embargo, los estudiantes del subgrupo A (los que utilizaron el equipo que ya estaba instalado en el salón de clases) calificaron como de regular el uso de comandos. Esto sugiere que el cañón y pantalla instalados ex profeso influenció de alguna manera la opinión de los estudiantes en cuanto al uso de los comandos. Según se vio en los datos cualitativos y cuantitativos, algunos de ellos declararon que los comandos eran algo complejos. En futuras aplicaciones del Raswin se utilizará un mínimo de comandos y se optará por el uso de scripts.

\section{DESARROLLO DE UNA GUÍA DE INTEGRACIÓN TECNOLÓGICA PARA EL MAESTRO}

Con base en los resultados cualitativos y cuantitativos obtenidos en el cuestionario de usabilidad aplicado en esta prueba, además de nuestra experiencia previa en el uso del RasMol y otros visualizadores, hemos desarrollado la siguiente guía para la integración de hardware y software de visualización molecular en el salón de clases:

- El diseño instruccional y la aplicación de hardware y software para la visualización molecular en el salón de clases debe ser multidisciplinar. Se debe tener la participación de al menos un pedagogo, el profesor titular de la materia (Bioquímica, Biología Molecular, etc.), además de un especialista y un técnico en computación, donde los representantes de cada disciplina aporten su experiencia y conocimientos. Para evitar conflictos interdisciplinarios, es necesario que el titular de la materia lleve el control de las participaciones de los expertos de cada área y recabar la información y comentarios generados.

- Prepare todo el equipo con tiempo. Puede llegar a ser desesperante (y en algunos casos frustrante) para los estudiantes el esperar varios minutos para montar la pantalla, encender el cañón, calibrarlo, etc.

- Prepare un "guión de actividades" de la clase con tiempo. ${ }^{40}$

- Practique previamente con el equipo, el software y el guión, para encontrar posibles fallas o errores y poder corregirlos, y adecuar las actividades al tiempo de la clase.

- En el caso de instalar un cañón de datos y una computadora, no siempre las condiciones de conexión a la energía eléctrica van a estar disponibles y ser las adecuadas en cualquier salón de clases. Cargue siempre un cable de extensión para energía eléctrica muy largo, de más de diez metros, para poder conectar el cañón de datos y la computadora a cierta distancia en el salón. De preferencia, utilice un regulador de voltaje, para evitar que se dañe el cañón y la computadora.

- Utilice un cañón de datos con una resolución nativa de al menos 1024 x 768 pixeles y una luminosidad de al menos 1800 lúmenes. Recuerde que pueden llegar a mostrarse estructuras moleculares complejas con mucho nivel de detalle que necesiten de mucha resolución de despliegue.

- Utilice una pantalla de calidad, con mucha rigidez, donde se pueda observar la proyección completamente. Una pantalla de inferior calidad o mal instalada podría doblarse y distorsionar la visualización molecular. Esto es crítico, ya que afectaría la percepción de la estructura y la conformación (el arreglo de los átomos de la molécula en el espacio) de las moléculas.
- Existen muchos programas de visualización molecular gratuitos, como el Raswin, que se pueden obtener de Internet. Estudios anteriores muestran que también programas similares como el Jmol y Chime se han utilizado con éxito en el salón de clases. La ventaja del Raswin es que puede correr prácticamente en cualquier computadora y puede ser programable.

- Proporcione a los estudiantes el programa de visualización molecular y los archivos en formato PDB (Protein Data Bank) que vaya a utilizar con ese programa visualizador, o al menos la URL de donde puedan ellos obtenerlo. Es posible que algunos de ellos tengan la inquietud de seguir explorando el software después.

- Para evitar teclear muchas instrucciones al momento de la clase, previa a la misma es posible programar en el Raswin una lista de instrucciones a manera de programa o scripts. ${ }^{24}$ Una explicación clara de esto se encuentra en la referencia anterior.

- Apóyese siempre con un asistente o un estudiante voluntario para manipular la estructura molecular en la computadora, mientras usted realiza explicaciones de la molécula.

- Establezca dinámicas de grupos para que la clase sea proactiva y que todos los estudiantes participen en el análisis de la visualización molecular. Puede ser posible que se formen equipos de estudiantes para analizar aspectos particulares de la estructura molecular y después cada grupo explicar sus conclusiones con el Raswin. Además, un representante de cada equipo tenga la oportunidad de manipular la visualización, o realizar interacciones con la misma.

- Recuerde poner en stand-by el cañón para dejar enfriar la lámpara el tiempo suficiente. Si lo desconecta inmediatamente después de utilizarlo puede dañar la lámpara irreparablemente.

- Cada vez que integre un nuevo software para la visualización molecular, le sugerimos que aplique un cuestionario de usabilidad, ya sea estándar o hecho a la medida como el mostrado en el Apéndice A, para obtener retroalimentación de los alumnos acerca de su uso.

- Cuando se integren nuevos visualizadores moleculares a la clase, se sugiere aplicar evaluaciones sumativas de usabilidad, por ejemplo la aplicación del cuestionario System Usability Scale (SUS), o como el mostrado en el Apéndice A.

\section{CONCLUSIONES}

Con respecto a las preguntas de investigación planteadas en el cuestionario del Anexo A se tienen las siguientes respuestas:

- ¿La calidad de proyección de las gráficas puede afectar la percepción general de aspectos importantes de las estructuras moleculares?

Los datos obtenidos del cuestionario indican que la calidad de proyección no es un factor determinante para observar las estructuras moleculares.

- ¿Los estudiantes encontrarán los comandos del Raswin fáciles de usar en clases?

No todos los estudiantes encontraron los comandos fáciles de usar. Sin embargo, los resultados indican que la calidad de la proyección puede influir positivamente en el uso de comandos.

- ¿El uso de un visualizador molecular como el Raswin motivará a los estudiantes a seguir utilizando el programa visualizador molecular en subsiguientes clases? 
Una respuesta completa a esta pregunta se dará en futuras evaluaciones de usabilidad. En este estudio se confirma que todos los estudiantes están totalmente de acuerdo en seguir utilizando programas como Raswin en subsiguientes clases.

Todos los datos cualitativos y cuantitativos obtenidos en el cuestionario de usabilidad, así como comentarios informales realizados durante el estudio, nos sirvieron de base para adecuar el hardware y software para utilizar en clase.

Es necesario seguir realizando más estudios de aplicación y pruebas de usabilidad de visualización científica, para de esta manera refinar y aumentar la guía propuesta. Es necesario también que otros profesores de química y algunos pedagogos también evalúen esos estudios y así obtener retroalimentación en cuanto a lineamientos pedagógicos-didácticos y contenidos. Asimismo, es necesario diseñar una nueva manera de evaluar conocimientos y competencias de los estudiantes utilizando visualización científica en el salón de clases.

\section{ANEXOA}

\section{Cuestionario de usabilidad}

Este cuestionario es para evaluar el sistema gráfico de los modelos moleculares y sus actividades en esta sesión, no se va a evaluar a ti. Favor de contestar las siguientes preguntas. En las preguntas de la 7 a la 12, marcar una "X" en la casilla correspondiente. Tus respuestas serán muy importantes para mejorar la aplicación de tecnología en el salón en el futuro.

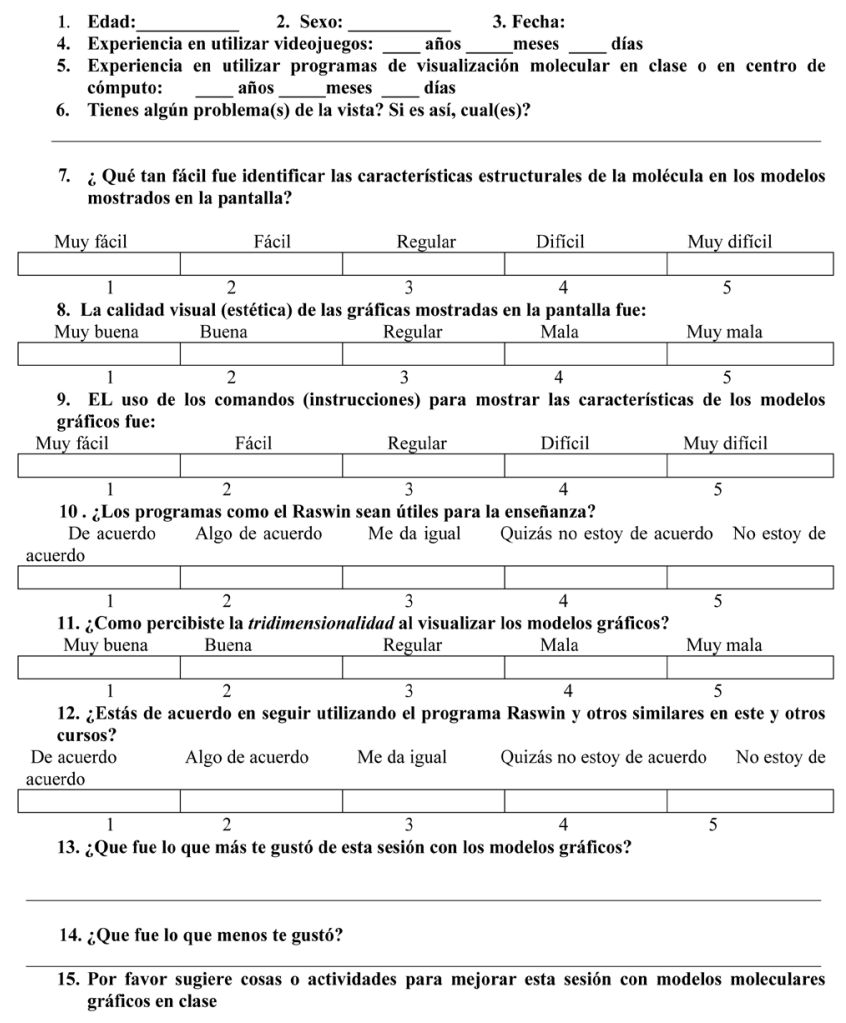

Muchas gracias por participar.

\section{REFERENCIAS}

1. Savec, V. F.; Vrtačnick, M.; Gilbert, J. K.; Perlaj, C.; Acta Chim. Slov. 2006, 53, 381.

2. Hardwicke, A. J.; Sch. Sci. Rev. 1995, 77, 59.

3. Petersen, Q. R.; J. Chem. Educ. 1970, 47, 24.

4. Jiménez, G.; Llitjós, A.; REEC 2006, 5, 111.

5. Jones, L.; Jordan, K.; Stillings, N.; Chem. Educ. Res. Pract. 2005, 6, 136.

6. Jones, M. B.; J. Chem. Educ. 2001, 78, 867.

7. Tasker, R. En Using multimedia to visualize the molecular world: educational theory into practice; Pienta, N.; Greenbowe, T.; Cooper, M., eds.; Prentice Hall: Upper Saddle River, 2004, ch. 16.

8. McCormick, B.; DeFanti, B.; Brown, R.; Computing Gaphics 1987, 21, 6.

9. Kozma, R. B.; Russell, J.; J. Res. Sci. Teach. 1997, 34, 949.

10. Williamson, V. M.; Abraham, M. R.; J. Res. Sci. Teach. 1995, 32, 521.

11. Hehre, W. J.; Nelson, J. E.; Introducing molecular modeling into the undergraduate chemistry curriculum, Wavefunction Inc: CA, 1997.

12. Shusterman, G. P.; Shusterman, A.; J. Chem. Educ. 1997, 74, 771.

13. Smith, S. G.; J. Chem. Educ. 1998, 75, 1080.

14. Jones, L. L.; UniServe Science News 1999, 14, 3.

15. Dori, Y. J.; Barak, M.; J. Ed. Tech. Soc. 2001, 4, 61.

16. Canning, D.; Cox, J. R.; Chem. Educ. Res. Pract. 2001, 2, 109.

17. Weiner, S. W.; Ferpovicz, C. P.; Dixon, D. W.; Harden, D. B.; Hobbs, D. S.; Gosnell, D. L.; J. Chem. Educ. 2000, 77, 401.

18. Cox, J. R.; J. Chem. Educ. 2000, 77, 1424.

19. Parrill, A. L.; Gervay, J.; J. Chem. Educ. 1997, 74, 1141.

20. Leon, D.; Uridil, S.; Miranda, J.; J. Chem. Educ. 1998, 75, 731.

21. Sayle, R. A.; Milner-Whilte, E. J.; Trends Biochem. Sci. 1995, 20, 374.

22. Bernstein, H. J.; Trends Biochem. Sci. 2000, 25, 453.

23. http://www.openRasMol.org, accedida en Julio 2007.

24. http://www.bernstein-plus-sons.com/software/RasMol_2.7.3/doc/esrasmol27.html, accedida en Septiembre 2007.

25. http://www.umass.edu/microbio/chime/pe_beta/pe/protexpl/frntdoor. html, accedida en Septiembre 2007.

26. http://expasy.org/spdbv/, accedida en Septiembre 2007.

27. http://kinemage.biochem.duke.edu/kinemage/magepage.php, accedida en Septiembre 2007.

28. Bottomley, S.; Chandler, D.; Morgan, E.; Helmerhorst, E.; Biochem. Mol. Biol. Educ. 2006, 34, 343.

29. http://www.wabri.org.au/jamvle/, accedida en Septiembre 2007.

30. Moll, A.; Hildebrandt, A.; Lenhof, H. P.; Kohlbacher, O.; Bioinformatics 2006, 22, 365 .

31. http://www.ballview.org/, accedida en Septiembre 2007.

32. Berman, H. M.; Westbrook, J.; Feng, Z.; Gilliland, G.; Bhat, T. N.; Weissig, H.; Shindyalov, I. N.; Bourne, P. E.; Nucleic Acids Res. 2000, $28,235$.

33. Preece, J.; Rogers, Y.; Sharp, H.; Interaction Design: Beyond Human Computer Interaction, $2^{\text {nd }}$ ed., Wiley: New York, 2002.

34. MacFarlane, S.; Sim, G.; Horton, M.; In Proceeding of the 2005 Conference on Interaction Design and Children, Boulder, USA, 2005.

35. Javahery, H.; Seffah, A.; Radhakrishnan, T.; Comms. of the ACM 2004, $47,11$.

36. http://www.ucc.ie/hfrg/resources/qfaq1.html, accedida en Diciembre 2006.

37. http://www.usabilitynet.org/trump/documents/Suschapt.doc, accedida en Septiembre 2007.

38. http://chemistry.gsu.edu/glactone/PDB/DNA_RNA/dna.html, accedida en Septiembre 2007.

39. http://av.bmbq.uma.es/pract/RasTut_e.html, accedida en Julio 2007.

40. Bauzá, B.; Cascudo, T.; Borén, H.; E-learning, Ediciones Anaya Multimedia: Madrid, 2003. 